\title{
On Medical Avatars in the Diagnosis and Treatment of Endometriosis
}

\author{
Alain L Fymat* \\ International Institute of Medicine and Science, California, USA \\ Received: 阱 June 08, 2018; Published: 眥 June 13, 2018 \\ *Corresponding author: Alain L Fymat, International Institute of Medicine and Science, California, USA, Tel: (760) 507-6862; \\ Email: alain.fymat@fiimas.org
}

\section{Editorial}

In the Hindu religion, an avatar is the incarnation of a god, a metamorphosis or a change, usually for the worse. More commonly, it is a frustrating event or an accident. By contrast, in the physical sciences and in engineering, it is a "model" that allows one to simulate numerically a physical phenomenon or instrument or an engineering device. Providing the model is a "good" representation of that phenomenon, instrument or device, it enables one to describe a certain reality. It also enables one to predict the instrument or device performance for any assumed governing parameters. Importantly, it obviates the need to physically construct that instrument or device for every plausible set of parameters, a hopeless waste of time, effort and resources. With the template provided by the model, all possible situations could be mimicked straightforwardly and their performance determined merely by changing the underlying parameters and running the template in a numerical processor (computer). In medicine, unfortunately, we have not yet arrived at "modeling" a human being in all its functions and dysfunctions. As a less than satisfactory alternative, and as a surrogate to the above modeling approach, we conduct clinical trials (whether using human or animal subjects), prescribe medications and, through a trial and error approach, hope to predict accurately the performance of the human organism, albeit in a limited way, and treat its malfunctions. It is indeed quite a fortunate circumstance that we have succeeded, at least in part, in that endeavor. Now, with the advent of the concept of an "organ on a chip", we have begun to design medical avatars that can help us diagnose more readily and more accurately some of the diseases affecting certain organs and, at least theoretically, provide treatment on a personalized basis. While we have just begun, we are far, perhaps hopelessly far, from being able to design an avatar that would model completely and with satisfactory accuracy the behavior and diseases of a whole human body. Since certain advances have been made in the design of an avatar for the endometrium, and prototype endometriums on a chip have been built and are undergoing testing and preclinical trials, I will limit here my considerations on avatars to that particular organ and its inflammatory state (endometriosis).
Endometriosis is the inflammation of the tissue lining the uterus (the endometrium) that has been aptly described as a "vine" through women's bodies causing pain and agony, heavy bleeding, discomfort, and infertility. It can spread to the bladder, the intestines, the ureter tubes and even the kidneys and other pelvic organs. When it reaches the fallopian tube, it squeezes it to such an extent that an egg cannot pass with the resulting infertility. The degree of scarring and the number and extent of the lesions do not correlate well with the severity of the symptoms. The cause is not well known because the disease has been ignored for decades and the associated research severely underfunded. In fact, even the basic question as to the mechanism by which the scar tissue migrates outside the uterus has not yet been answered.

Several theories have been propounded as to the cause of the disease:

I. The so-called Sampson's "retrograde menstruation" hypothesis, including its three variants

a) The "seed" or rogue endometrial and stem cells in the menstrual fluid that linger behind, implant wherever they are, causing inflammation that leads to scarring and adhesions;

b) The "soil" or abdominal environment where those cells implant and spread; or

c) The "soil changing the seed" combination, a combined state between (a) and (b) above;

II. The Osteen's "toxic hypothesis" where environmental toxins (especially the industrial byproducts dioxin and dioxin-like chemicals such as polychlorinated biphenyls (PCBs)) play a role, at least in some cases;

III. The "multicausal" hypothesis where endometriosis is the likely result of many causes and manifestations, some of which being unspecified;

IV. The endometrial "micro biome" hypothesis; 
V. The "immune dysfunction" hypothesis; and

VI. Genetics, which may account for approximately 50\%, the other causative factors (described above) being not fully understood.

Diagnosis currently requires a surgical procedure (a laparoscopic test), but it takes place several years (typically seven) after the onset of the disease. In the meantime, the ravages of the disease have gone unabated. At present, there is no known simple biochemical marker in a blood, saliva or urine test. Current treatments consist of

a. medication;

b. Off-label botox (botulinum toxin) injections in the pelvic floor to relax muscle spasms, alter chemicals involved in pain signaling, and reverse the brain's central sensitization to pain; and

c. Surgery, which unfortunately may have to be repeated approximately every seven years.

However, hope is on the horizon along two different lines. First, three microRNAs (mRNA) have been found to be more prevalent in endometriosis patients. Recall that mRNAs are short, non coding sequences of RNA that regulate gene expression and are shed by tissues. They can be used as the basis for a diagnostic saliva test and may also be used to assess whether a prescribed drug is effective both initially, during the course of treatment and even after treatment would long have been discontinued. Next, a skin biopsy could be "reverse engineered" in developmental time to become induced pluripotent stem cells. A preferable alternative might be an endometrial biopsy. Such cells could be used to seed a "micro organ" on a tablet electronic circuit board representing the patient's body. This would be the patient's medical or "organ on a chip" avatar. In this case, the avatar is a miniature female reproductive system, complete with micro ovaries, fallopian tube, uterus, cervix, and liver.

The avatar consists of three superimposed elements:

a. a base plate consisting of an integrated electronic board that contains microfluidic pumps/vessels and is connected to a computer means;

b. an intermediary plate, the endometrium platform proper, which represents the interface between the electronic elements below and the biological elements above; and

c. an upper plate that includes living cells of endometrium, ovary (ies), bone marrow, the gut and immune system, the liver and immune system, and the means to make these several organs interact with each other, all of which being harbored in dime-sized vessels. The endometrium element consists of polymer hydrogel scaffolding that supports several layers of endometrial tissue cell types. The number of such mini-organ systems is unclear but is currently envisioned not to exceed ten.

Synthetic blood flows through micro channels that connect the organs, carrying hormones, nutrients, and growth and immune factors between them. The avatar can even functionally be endowed with a monthly cycle but not menstrual periods with the attendant bleeding. It is important to note that this type of avatar is for the particular patient under analysis and investigation (along the precepts of personalized or customized medicine). It can be used to test the potential interaction of that patient with proposed drugs to assess their potential safety and efficacy. Various hormones and inflammatory cues could be applied to the tissues. The avatar could be tested during a full menstrual cycle, collecting and analyzing data from each represented organ. A proof of concept of this device has already been built at Northwestern University School of Medicine. However, its full development and clinical application are years in the future. More modestly, rather than developing an individualized avatar for each patient, it has also been proposed to stratify endometriosis patients by several molecular markers and develop avatars for such patient categories. Notwithstanding the novelty and originality of medical avatars, it must be recognized that they suffer from the similar limitations as their counterparts in physics, engineering and science that have been discussed in the introductory paragraph. The difficulties are multi-fold. First and foremost, as set up, the avatar must be a good model of the underlying biology which, unfortunately, is not well understood. Thus, the number and type of mimicking organs is uncertain. In addition, and in contrast with the physical, engineering and science models, the avatar is a "living", dynamic system. We are years away from routine diagnostic and therapeutic treatment of endometriosis, but the future is hopeful and medical avatars may bring us closer to that date.
This work is licensed under Creative Commons Attribution 4.0 License

To Submit Your Article Click Here: Submit Article

DOI: $10.32474 /$ IGWHC.2018.02.000133

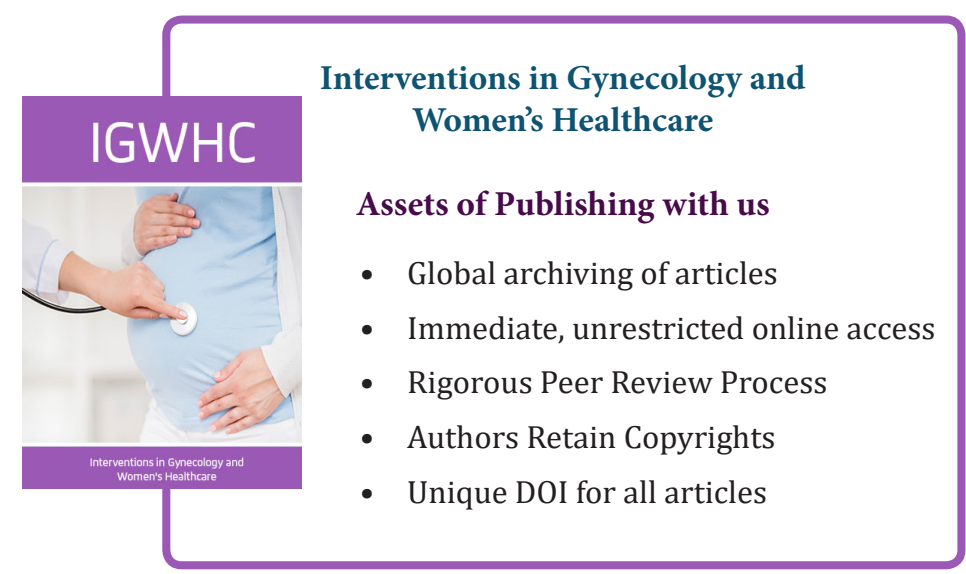

Interventions in Gynecology and Women's Healthcare

Assets of Publishing with us

- Global archiving of articles

- Immediate, unrestricted online access

- Rigorous Peer Review Process

- Authors Retain Copyrights

- Unique DOI for all articles 